2-23-2015

\title{
Non-Suicidal Self-Injury in a Large Online Sample of Transgender Adults
}

lore m. dickey

Sari L. Reisner

Cindy L. Juntunen

University of North Dakota, cl.juntunen@UND.edu

How does access to this work benefit you? Let us know!

Follow this and additional works at: https://commons.und.edu/ehb-fac

Part of the Health and Physical Education Commons

\section{Recommended Citation}

lore m. dickey, Sari L. Reisner, and Cindy L. Juntunen. "Non-Suicidal Self-Injury in a Large Online Sample of Transgender Adults" (2015). Education, Health \& Behavior Studies Faculty Publications. 8.

https://commons.und.edu/ehb-fac/8

This Article is brought to you for free and open access by the Department of Education, Health \& Behavior Studies at UND Scholarly Commons. It has been accepted for inclusion in Education, Health \& Behavior Studies Faculty Publications by an authorized administrator of UND Scholarly Commons. For more information, please contact und.commons@library.und.edu. 


\title{
Non-Suicidal Self-Injury in a Large Online Sample of Transgender Adults
}

\author{
lore m. dickey \\ University of North Dakota and Louisiana Tech University;
}

Sari L. Reisner

Harvard School of Public Health and The Fenway Institute, Fenway Health, Boston, Massachusetts

\section{Cindy Lee Juntunen}

University of North Dakota

\begin{abstract}
Non-suicidal self-injury (NSSI) has been increasing in clinical as well as nonclinical populations in recent years. There are few published reports examining lifetime occurrence of this behavior in transgender and gender nonconforming (TGNC) people. An online survey was conducted with self-identified TGNC adults $(n=773)$ in the United States over the course of 6 months in 2009. The mean age for the sample was 40.4 years $(S D=13.9)$. Most participants identified on the trans masculine spectrum (female-to-male or FTM; 52.0\%), 33.9\% identified on the trans feminine spectrum (male-to-female or MTF), and $8.0 \%$ identified as genderqueer. Participants completed the Body Investment Scale, the Depression, Anxiety, and Stress Scales, and the Inventory of Statements about Self-Injury. Results indicated that $41.9 \%$ of participants had a lifetime history of NSSI. Scores on the subscales of Protection and Feeling from the Body Investment Scale were found to be statistically predictive of NSSI. These findings shed new light on the lifetime prevalence of NSSI in this online TGNC respondent sample. Practice implications are discussed for mental health professionals who work with TGNC clients in addition to research recommendations. (PsycINFO Database Record (c) 2016 APA, all rights reserved)
\end{abstract}

Transgender is an umbrella term used to describe people whose gender identity or expression does not conform to that typically associated with the sex they were assigned at birth (Lev, 2004). Clients with a transgender or gender nonconforming (TGNC) history seek counseling for a variety of reasons. This might include basic issues of adjustment to stressors in daily life, family and employment concerns, and support to engage in transition or gender affirmation processes. This article presents information from an online study examining the lifetime prevalence of non-suicidal self-injury (NSSI) in a TGNC community sample and offers implications for practice and research with TGNC people.

TGNC individuals have been shown to disproportionately experience stressors across the life course, including in multiple domains (family, work, school) and at multiple levels (interpersonal, intrapersonal, institutional; Grant et al., 2011). Exposure to myriad of interacting stressors including social (discrimination, stigma, violence), psychological (internalized transphobia, depression, anxiety, body image concerns), and biological (hormones, surgeries, lack of access to and utilization of preventive care/screening) may place TGNC people at high risk for negative mental health outcomes. In addition, there is evidence to suggest that TGNC individuals are at risk of managing stress with potentially problematic, health-damaging, or 
injurious coping behaviors (Hepp, Kraemer, Schnyder, Miller, \& Delsignore, 2005), such as NSSI.

NSSI is a behavioral concern in which people deliberately harm themselves without lethal intent (Klonsky \& Muehlenkamp, 2007; Nock, 2009). In the United States, between 1\% and $4 \%$ of adults and $13 \%$ to $23 \%$ of adolescents report a lifetime history of NSSI (see Jacobson \& Gould, 2007, for review). Estimates of prevalence of NSSI vary considerably depending on the definition and measurement, including timeframe of self-reported NSSI, as well as the population sampled and study design being used (Favazza, 1998; Muehlenkamp, 2005). Behaviors associated with NSSI include cutting, burning, severe scratching, hitting, and interfering with wound healing (Favazza, 1998; Muehlenkamp, 2005); excluded from NSSI are culturally sanctioned body modification procedures, such as tattooing or piercing as per previous NSSI research (Nock, 2009). We also exclude TGNC-related body modification in our operationalization of NSSI behaviors for transgender people. TGNC-related body modifications include medically sanctioned procedures, such as chest or genital surgery. Because these modifications are not self-administered, they cannot be considered NSSI. Also excluded from consideration are suicidal behaviors (i.e., behaviors with lethal intent). In the general public, estimates of NSSI have recently been shown to be similar between men and women (Klonsky \& Muehlenkamp, 2007). The major difference in NSSI between men and women appears to be the manner in which they hurt themselves. According to Klonsky and Muehlenkamp (2007), "women [are] more likely to engage in cutting [and] men are more likely to burn or hit themselves" (p. 1047).

There have been no large empirical studies in the peer-reviewed research literature of NSSI among TGNC communities and little application of existing theoretical frameworks to understand experiences of NSSI among TGNC individuals. In addition, most research either fails to ask about gender identity or the sample of TGNC people is too small to make any withingroup statistical comparisons to identify correlates and risk factors for NSSI among TGNC respondents (Institute of Medicine, 2011). In the general population, the strongest correlates of lifetime NSSI are psychiatric disorders and other co-occurring presenting mental health concerns, including but not limited to depression and anxiety (Klonsky \& Olino, 2008; Muehlenkamp, 2005; Nock, 2009). Evidence shows that the prevalence of depression, anxiety, and other co-occurring mental health concerns is high among TGNC people. For example, the prevalence of lifetime depression among TGNC adult samples in the published peer-reviewed literature is as high as $60 \%$ (e.g., Clements-Nolle, Marx, \& Katz, 2006). Given the link between NSSI and other mental health concerns, and the high prevalence of depression among TGNC people, it is possible that lifetime prevalence of NSSI is also high among TGNC adults.

Body image concerns have also been documented among TGNC people and may be a risk factor for NSSI (Vocks, Stahn, Loenser, \& Legenbauer, 2009). In the general population, NSSI usually begins in early adolescence (Klonsky \& Muehlenkamp, 2007) and may persist behaviorally for many years (Muehlenkamp, 2005). For TGNC people, the biological pubertal changes that occur during adolescence may increase psychological distress associated with gender identity and 
expression, including body image, and may be a stress trigger relevant to understanding the timing and onset of NSSI behaviors across the life course.

This study was conducted to address important gaps in the research literature about lifetime NSSI among TGNC people and to contribute to greater understanding of the unique needs of TGNC individuals, as well as the practitioners and health care providers who serve them, who have historically been overlooked in psychology (Sánchez \& Vilain, 2009). The overarching aims of the study were to (a) understand the experience of NSSI among TGNC people, including documenting the self-reported lifetime prevalence and type of NSSI behaviors in a large online sample; and (b) examine the co-occurring mental health concerns among TGNC people with a history of NSSI.

\section{Method}

\section{Participants}

A convenience sample of 773 self-identified TGNC adults in the United States completed a cross-sectional quantitative online survey in 2009. Participation was limited to those living in the United States as self-reported on the demographic questionnaire. The majority of participants in the study identified on the trans masculine spectrum (52.0\%; female-to-male or FTM) followed by trans feminine spectrum (33.9\%; male-to-female or MTF), genderqueer or TGNC (8.0\%), and participants with another gender identity that is not on the gender binary (4.9\%). Other demographic data for the sample included the following: $72.1 \%$ of the participants were White (non-Hispanic), $54.2 \%$ of participants had a college or graduate degree, and $45.8 \%$ made less than $\$ 25,000$ a year. Characteristics of the study sample are presented in Table 1.

Table 1

Demographic Characteristics of the Study Sample

\begin{tabular}{|c|c|c|c|}
\hline Characteristics & $\begin{array}{c}\text { NSSI } \\
(n=324)\end{array}$ & $\begin{array}{c}\text { No NSSI } \\
(n=449)\end{array}$ & $\begin{array}{c}\text { Total sample } \\
(n=773)\end{array}$ \\
\hline \multicolumn{4}{|l|}{ Age, $M(S D)$} \\
\hline Current age & $34.5(12.5)$ & 44.7 (13.2) & $40.4(13.9)$ \\
\hline Age first NSSI & $13.6(7.0)$ & - & - \\
\hline Age most recent NSSI & $27.3(11.6)$ & - & - \\
\hline \multicolumn{4}{|l|}{ Gender, $n(\%)$} \\
\hline Genderqueer/transgender & $32(9.9)$ & $30(6.7)$ & $62(8.0)$ \\
\hline Male spectrum (female-to-male) & $183(56.5)$ & $219(48.8)$ & $402(52.0)$ \\
\hline Female spectrum (male-to-female) & $89(27.5)$ & $173(38.5)$ & $262(33.9)$ \\
\hline Other nonbinary gender & $20(6.2)$ & $27(6.0)$ & $47(6.1)$ \\
\hline \multicolumn{4}{|l|}{ Race/ethnicity, $n(\%)$} \\
\hline Racial/ethnic minority & $49(15.1)$ & $42(9.4)$ & $91(11.8)$ \\
\hline White & $214(66.0)$ & $343(76.4)$ & $557(72.1)$ \\
\hline Missing race/ethnicity & $61(18.8)$ & $64(14.3)$ & $125(16.2)$ \\
\hline \multicolumn{4}{|l|}{ Sexual orientation, $n$ (\%) } \\
\hline Straight & $55(17.0)$ & $153(34.1)$ & $208(26.9)$ \\
\hline Lesbian/gay & 47 (14.5) & $81(18.0)$ & $128(16.6)$ \\
\hline Bisexual & $93(28.7)$ & $113(25.2)$ & $206(26.6)$ \\
\hline Asexual & $10(3.1)$ & $16(3.6)$ & $26(3.4)$ \\
\hline Queer & 96 (29.6) & $58(12.9)$ & 154 (19.9) \\
\hline Other nonbinary identity & $22(6.8)$ & $25(5.6)$ & $47(6.1)$ \\
\hline \multicolumn{4}{|l|}{ Socioeconomic, $n$ (\%) } \\
\hline \multicolumn{4}{|l|}{ Education } \\
\hline High school or below & $27(8.4)$ & $28(6.2)$ & $55(7.1)$ \\
\hline Some college, trade degree & $134(41.3)$ & $163(36.3)$ & $297(38.4)$ \\
\hline College degree & $86(26.5)$ & $143(31.8)$ & $229(29.6)$ \\
\hline Graduate degree & $75(23.2)$ & $115(25.6)$ & $190(24.6)$ \\
\hline \multicolumn{4}{|l|}{ Income, $n(\%)$} \\
\hline$<\$ 12,000$ & $113(34.9)$ & $68(15.1)$ & $181(23.4)$ \\
\hline$\$ 12,000$ to $\$ 25,000$ & $88(27.2)$ & $85(18.9)$ & $173(22.4)$ \\
\hline$\$ 25,001$ to $\$ 50,000$ & $69(21.3)$ & $109(24.3)$ & $178(23.0)$ \\
\hline$\$ 50,001$ to $\$ 100,000$ & 44 (13.6) & $126(28.1)$ & $170(22.0)$ \\
\hline Greater than $\$ 100,000$ & $9(2.8)$ & $55(12.2)$ & $64(8.3)$ \\
\hline
\end{tabular}




\section{Measures}

\section{Sociodemographic characteristics}

Participants were asked to complete several questions that addressed demographic characteristics. Participants were asked their age, gender, income, education level, sexual orientation, and racial/ethnic identity. Because of an error, an early version of the survey did not include a question about racial and ethnic identity. This error was corrected, though it is responsible for the lack of data on this characteristic for 102 participants. To account for any bias introduced because of missing data for race/ethnicity, participants were classified as racial/ethnic minority (people of color), White non-Hispanic, or missing race/ethnicity. Participants could write in their self-identified gender identity. The first author and a colleague coded the data from this question into nine categories. Because several categories had very small numbers, they were further recoded for ease of statistical analysis as follows: genderqueer or TGNC, trans masculine spectrum (FTM), trans feminine spectrum (MTF), and other gender (nonbinary). The authors recognize the challenges associated with this categorization of the data and acknowledge the importance of autonomy as it relates to one's gender identity. Participants were asked "Have you begun to transition?" Transition (for this question) was not defined, rather participants made their own meaning of this term.

\section{Depression Anxiety Stress Scales (DASS-21)}

The DASS-21 is a psychometrically validated short form of the full DASS (a 42-item scale; Henry \& Crawford, 2005; Lovibond \& Lovibond, 1995). The DASS-21 contains 21 items and is a self-report measure of depression, anxiety, and stress experienced in the past week. Sample items: "I found it hard to wind down," "I felt down-hearted and blue," and "I was intolerant of anything that kept me from getting on with what I was doing." Responses were scored on a 4-point Likert scale ranging from $0=$ "did not apply to me at all" to $3=$ "applied to me very much, or most of the time." Scores were summed so that high scores indicate elevated levels of depression, anxiety, and stress. Subscale scores on the DASS-21, which were used in this study, range from 0 to 42 (scores on the DASS-21 are doubled prior to interpretation, as recommended by the scale authors). Severity ratings for each of the subscales from a normative sample indicate that Depression scores between 10 and 13 are mild and between 14 and 20 are moderate. Anxiety scores between 8 and 9 are mild and between 10 and 14 are moderate. Stress scores from 15 to 18 are mild and from 19 to 25 are moderate. In the current study, the overall scale evidenced good reliability (Cronbach's alpha $=.93$ ), as did the subscales (Cronbach's alpha $=.92$ for depression, 0.83 for anxiety, and 0.85 for stress). In addition to showing acceptable reliability (Cronbach's alpha $=.81$ for depression, 0.73 for anxiety, and 0.81 for stress; Lovibond \& Lovibond, 1995), the DASS-21 shows convergent validity when compared with the Beck Depression and Anxiety Inventories; $r=.74$ and 0.81, respectively (Henry \& Crawford, 2005). 


\section{Body Investment Scale (BIS)}

The BIS is a 24-item self-report instrument that is designed to measure a person's experience, feelings, and attitudes about his or her body (Orbach \& Mikulincer, 1998). The BIS has been shown to have good psychometric properties with acceptable reliability (Cronbach's alpha ranging from 0.80 to 0.95 ) and strong validity when compared with the Multi-Attitude Suicidal Tendencies Scale (MAST; subscale correlations ranged from $r=|.09|$ to $|.56|$; Orbach \& Mikulincer, 1998). Sample items: "I believe that caring for my body will improve my wellbeing," "I don't like it when people touch me," "I am satisfied with my appearance," and "I feel anger toward my body." Responses are scored on a 5-point Likert scale from $1=$ "do not agree at all" to 5 = "strongly agree." The BIS contains four subscales, which were used in this study, with 6 items each: (a) body image feelings and attitudes, (b) comfort in touch, (c) body care, and (d) body protection. Scores on the subscales range from 6 to 30 . The overall scale reliability was high in the current study (Cronbach's alpha $=.89$ ), as well as for the subscales (Cronbach's alpha ranging from 0.67 to 0.91 ). High scores on each subscale indicate that the individual has a strong investment in their body. Low scores indicate little investment in one's body.

\section{Non-suicidal Self-Injury and Inventory of Statements About Self-Injury (ISAS)}

The last question on the demographic form asked about a history of NSSI. Lifetime self-reported NSSI was queried by asking participants "Have you ever engaged in self-injurious behavior (e.g., cutting, burning, hitting, pulling hair, etc.)?" Response options were $1=$ "yes" and 2 = "no".

The ISAS was developed to assess lifetime frequency and functions of NSSI (Klonsky \& Glenn, 2009). The ISAS consists of two parts. The first part is a checklist of lifetime NSSI in which the participants indicate how many times they have participated in 12 NSSI behaviors. The first part also asks for a historical record of the presence of NSSI in a person's life. The second part of the survey is a listing of 39 functions of NSSI. For the purposes of this article, data are reported from the first part of the ISAS. This includes information about the history of NSSI and the types of behaviors, but not the functions. Latimer and colleagues (2013) used a Rasch Model to test the unidimensionality of the first part of the ISAS, which showed good fit, $\chi^{2}=38.3(d f=24), p=$ .03. In addition, ISAS has good reliability (Cronbach's alpha $=.83$; Latimer, Meade, \& Tennant, 2013).

\section{Procedures}

The online survey was designed in SurveyGizmo, an online research tool. To control for order effects, the DASS-21 and the BIS were presented in random order. The demographic questionnaire followed these scales. The last question in the demographic questionnaire asked whether participants had a history of self-injurious behavior. Participants who answered no were thanked for their participation and provided with the contact information of the first author. If they answered yes, they were asked to complete the ISAS.

The survey consisted of five parts: (a) informed consent, (b) survey qualification (i.e., confirmation of eligibility), (c) survey measures, (d) demographic data, and (e) the ISAS. The introductory page gave potential participants the information necessary to complete informed 
consent. Participants were offered no compensation for participation; however, they were given the opportunity to be included in a raffle for twenty $\$ 100$ cash prizes.

\section{Recruitment}

The name of the study was "Mental Health in the Transgender Community." This title was chosen with the hope that participants would not self-select out of the study on learning that the primary mental health concern was lifetime NSSI behaviors. Participants were recruited using purposive sampling strategies from three sources. The first source was social media such as Yahoo! Groups and Facebook. Messages were sent to 47 Yahoo! Groups relevant to the TGNC community. The first author created an event on Facebook to recruit study participants. The second source for participants was through gender clinics and gender specialists throughout the United States. Providers who agreed to assist with the recruitment were sent paper copies of the survey, a supply of postcards and business cards created to promote this research project, and a form to order additional materials. Finally, research participants were recruited at four conferences for the TGNC community. At two of the conferences, a vendor table was secured for help in promoting the research project. The first author staffed this table, provided materials to prospective participants, and answered questions about the study. At the other conferences, informational postcards and business cards were made available to participants in the lobby areas. Regardless of recruitment source, all participants accessed the study via a link to the Internet-based survey.

The study was institutional review board (IRB)-approved at the University of North Dakota. This was an anonymous survey, in which neither personal identifying information nor IP addresses were collected. Participants who agreed to participate in the survey acknowledged having read the informed consent and thereby agreed to participate in the research project.

\section{Requirements for participation}

The first two questions of the survey were used to verify whether participants met the inclusion criteria for the study. The questions asked (a) whether the person identified as "transgender (e.g., transgender, transsexual, cross dresser, drag king, drag queen, gender queer, etc.)," and (b) whether they were at least 18 years of age. Participants who answered no to either question were directed to a page that thanked them for their interest and provided contact information of the first author. We specifically used broad terminology related to gender identity in an effort to capture participants from a range of gender identities.

\section{Data Analysis}

\section{Primary outcome: NSSI}

The primary outcome variable was a binary indicator of self-reported lifetime history of NSSI. Those who reported no history of NSSI were considered to not have NSSI histories.

\section{General statistical procedures}

All data collected from this survey were analyzed using SPSS v21 statistical software, with significance predetermined at the alpha 0.05 level. Descriptive statistics were obtained for all 
variables, including the frequencies of binary and categorical variables and the distribution of all scale scores. The general analytic strategy was to compare respondents who reported lifetime NSSI with those who did not. Independent $t$ tests (with tests for equal or unequal variances) were estimated to test for differences between depression, anxiety, stress, and body investment based on the participants' NSSI history. In addition, a discriminant function analysis was performed using canonical linear discriminant functions to statistically predict group membership (i.e., classifying respondents by history of NSSI; Huberty, 1984) for the full sample and then for FTMs and MTFs.

\section{Results}

Overall, $41.9 \%$ of the sample $(n=324)$ reported a history of NSSI. Participants who reported NSSI had a mean age of 34.5 years $(S D=12.5)$ and reported first engaging in NSSI age a mean age of 13.6 years $(S D=7.0)$, and having engaged in NSSI most recently at a mean age of 27.3 years $(S D=11.6)$. Table 2 shows a breakdown, by gender or sexual orientation, of those with a history of NSSI. Table 3 presents a breakdown of NSSI behaviors. Among those who reported lifetime NSSI in this sample, the most common type of NSSI was cutting (66.4\%). Table 3 also shows a breakdown of participants based on gender or sexual orientation and the type of NSSI in which a person engaged. Participants who identified as genderqueer (not MTF, FTM, or other nonbinary), and those who identified as queer (not straight, lesbian, gay, bisexual, or asexual) tended to report levels of NSSI that were higher than the average for the full sample.

Table 2

Breakdown of Participants by Gender or Sexual Orientation Who Reported a History of Non-suicidal Self-Injury (NSSI)

\begin{tabular}{lc}
\hline \multicolumn{1}{c}{ Variable } & $\begin{array}{c}\text { History of NSSI, } \\
n(\%)\end{array}$ \\
\hline Gender & \\
Genderqueer/transgender & $32(51.6)$ \\
Male spectrum (female-to-male) & $183(45.5)$ \\
Other nonbinary & $20(42.6)$ \\
Female spectrum (male-to-female) & $89(34.0)$ \\
Sexual orientation & \\
Queer & $96(62.3)$ \\
Other sexual orientation & $22(46.8)$ \\
Bisexual & $93(45.1)$ \\
Asexual & $10(38.5)$ \\
Lesbian/gay & $47(36.7)$ \\
Heterosexual & $55(26.4)$ \\
\hline
\end{tabular}

Breakdown of Participants by Gender or Sexual Orientation Who Reported a History of Nonsuicidal Self-Injury (NSSI) 
Table 3

Breakdown of Non-Suicidal Self-Injury (NSSI) Behaviors Reported by Percentage of Participants With Lifetime NSSI Histories

\begin{tabular}{|c|c|c|c|c|c|c|c|c|c|c|c|}
\hline \multirow[b]{2}{*}{ NSSI behavior } & \multirow[b]{2}{*}{$\begin{array}{c}\text { History of NSSI } \\
(n=324)\end{array}$} & \multicolumn{4}{|c|}{ Gender } & \multicolumn{6}{|c|}{ Sexual orientation } \\
\hline & & $\begin{array}{l}\text { Female-to-male } \\
\quad(n=183)\end{array}$ & $\begin{array}{l}\text { Male-to-female } \\
\quad(n=89)\end{array}$ & $\begin{array}{l}\text { Genderqueer } \\
(n=32)\end{array}$ & $\begin{array}{c}\text { Other } \\
(n=16)\end{array}$ & $\begin{array}{c}\text { Heterosexual } \\
\quad(n=55)\end{array}$ & $\begin{array}{c}\text { Lesbian/gay } \\
(n=47)\end{array}$ & $\begin{array}{l}\text { Bisexual } \\
(n=93)\end{array}$ & $\begin{array}{l}\text { Asexual } \\
(n=10)\end{array}$ & $\begin{array}{c}\text { Queer } \\
(n=96)\end{array}$ & $\begin{array}{c}\text { Other } \\
(n=22)\end{array}$ \\
\hline Cutting & 66.4 & 73.2 & 52.8 & 75.0 & 62.5 & 67.3 & 66.0 & 59.6 & 60.0 & 70.8 & 77.3 \\
\hline Banging or hitting self & 55.2 & 56.8 & 55.1 & 43.8 & 75.0 & 49.1 & 53.2 & 62.4 & 50.0 & 57.3 & 54.5 \\
\hline Punching & 50.6 & 51.2 & 44.9 & 59.4 & 62.5 & 49.1 & 42.6 & 52.7 & 60.0 & 53.1 & 63.6 \\
\hline Interfere with wound healing & 35.8 & 34.4 & 33.7 & 43.8 & 56.3 & 32.7 & 29.8 & 36.6 & 40.0 & 40.6 & 36.4 \\
\hline Severe scratching & 34.9 & 32.9 & 32.6 & 50.0 & 50.0 & 25.5 & 23.4 & 40.9 & 30.0 & 40.6 & 45.5 \\
\hline Burning & 33.0 & 36.6 & 25.8 & 31.3 & 43.8 & 30.9 & 31.9 & 34.4 & 10.0 & 35.4 & 45.5 \\
\hline Pulling hair & 32.4 & 31.7 & 30.3 & 34.4 & 56.3 & 23.6 & 29.8 & 36.6 & 30.0 & 36.5 & 40.1 \\
\hline Biting & 29.6 & 25.7 & 30.3 & 40.6 & 56.2 & 16.4 & 19.1 & 31.2 & 20.0 & 39.6 & 40.1 \\
\hline Sticking self with needles & 24.7 & 19.1 & 33.7 & 21.9 & 50.0 & 18.2 & 23.4 & 24.7 & 40.0 & 25.0 & 40.1 \\
\hline Carving & 23.5 & 29.5 & 14.6 & 15.6 & 25.0 & 40.0 & 23.4 & 24.7 & 20.0 & 20.8 & 40.1 \\
\hline Rub skin against rough surface & 21.0 & 21.3 & 20.2 & 18.8 & 31.3 & 14.5 & 21.3 & 25.8 & 10.0 & 20.8 & 27.3 \\
\hline Swallow dangerous substances & 17.6 & 19.7 & 16.9 & 9.4 & 18.8 & 16.4 & 10.6 & 23.7 & 30.0 & 14.6 & 22.7 \\
\hline
\end{tabular}

Note. Values in boldface type indicate that this group reports engaging in this method of NSSI at rates $5 \%$ greater than the average of the full sample. These differences were tested statistically.

Breakdown of Non-Suicidal Self-Injury (NSSI) Behaviors Reported by Percentage of Participants With Lifetime NSSI Histories

\section{DASS-21 and NSSI}

Participants showed mild levels of depression compared with normative data $(M=12.3, S D=$ 10.3). For both anxiety and stress, the participant results were on the high end of normal scores (anxiety $M=7.6, S D=7.6$; stress $M=14, S D=8.5$ ). Participants with a history of NSSI showed statistically significantly higher levels of depression, anxiety, and stress at the alpha 0.05 level as shown in Table 4.

Table 4

Depression Anxiety Stress Scales (DASS) Results by

Non-Suicidal Self-Injury (NSSI) Status $(n=773)$

\begin{tabular}{lllc}
\hline & \multicolumn{3}{c}{$M(S D)$} \\
\cline { 2 - 4 } \multicolumn{1}{c}{ Status } & Depression & Anxiety & \multicolumn{1}{c}{ Stress } \\
\hline Full sample $(n=773)$ & $12.3(10.3)$ & $7.6(7.6)$ & $14.0(8.5)$ \\
History of NSSI & $14.8(10.8)^{\mathrm{a}}$ & $9.6(8.2)^{\mathrm{b}}$ & $16.6(8.6)^{\mathrm{c}}$ \\
No history of NSSI & $10.5(8.5)$ & $6.1(6.7)$ & $12.2(8.00)$ \\
\hline
\end{tabular}

a This mean was statistically significantly different than those without a history of NSSI $(t$-test statistic $=5.8, p<.01$, Cohen's $d=0.42)$. b This mean was statistically significantly different than those without a history of NSSI ( $t$-test statistic $=6.2, p<.01$, Cohen's $d=0.45$ ). ' This mean was statistically significantly different than those without a history of NSSI ( $t$-test statistic $=7.4, p<.01$, Cohen's $d=0.59)$.

Depression Anxiety Stress Scales (DASS) Results by Non-Suicidal Self-Injury (NSSI) Status $(n=$ 773)

\section{BIS and NSSI}

We examined whether BIS scores were associated with a lifetime history of NSSI. High scores on the BIS, and on each BIS subscale, indicate that the individual has a strong investment in their body. Participants with a history of NSSI had statistically significantly lower scores on each of the subscales compared with participants without a history of NSSI (Table 5). 
Table 5

Body Investment Scale (BIS) Results by Non-Suicidal Self-Injury (NSSI) Status $(n=773)$

\begin{tabular}{lcccc}
\hline & \multicolumn{4}{c}{$M(S D)$} \\
\cline { 2 - 5 } & Feeling & Touch & Care & Protection \\
\hline Full sample & $17.1(6.0)$ & $21.1 .(5.0)$ & $23.4(3.5)$ & $22.4(3.9)$ \\
History of NSSI & $15.1(5.7)^{\mathrm{a}}$ & $19.9(5.0)^{\mathrm{b}}$ & $22.5(3.6)^{\mathrm{e}}$ & $20.6(4.0)^{\mathrm{d}}$ \\
No history of NSSI & $18.5(5.8)$ & $21.9(4.8)$ & $24.1(3.3)$ & $23.7(3.2)$ \\
\hline
\end{tabular}

"This mean was statistically significantly different than those without a history of NSSI ( $t$-test statistic $=-8.2, p<.01$, Cohen's $d=$ -0.60 ). ${ }^{b}$ This mean was statistically significantly different than those without a history of NSSI ( $t$-test statistic $=-5.7, p<.01$, Cohen's $d=$ -0.42 ). "This mean was statistically significantly different than those without a history of NSSI ( $t$-test statistic $=-6.6, p<.01$, Cohen's $d=-0.48$ ). ${ }^{d}$ This mean was statistically significantly different than those without a history of NSSI ( $t$-test statistic $=-11.7, p<.01$, Cohen's $d=-0.85$ ).

Body Investment Scale (BIS) Results by Non-Suicidal Self-Injury (NSSI) Status $(n=773)$

A discriminant function analysis was conducted to determine whether the BIS subscales were statistically predictive of lifetime NSSI. Results indicate that the Body Protection and the Body Image Feelings and Attitudes subscales were both statistically predictive of ever engaging in NSSI, Wilks' $\Lambda=0.815, \chi^{2}(4, n=773)=156.98, p<.001$. The discriminant analysis was then restricted to FTM, $\Lambda=0.824, \chi^{2}(4, n=402)=77.03, p<.01$, and to MTF, $\Lambda=0.834, \chi^{2}(4, n=$ $262)=46.88, p<.001$, participants, and the results remained significant, though different subscales were statistically predictive of NSSI history by gender identity (see Table 6 for a breakdown of these differences).

Table 6

Standardized Coefficients and Correlations of

Predictor Variables

\begin{tabular}{|c|c|c|c|c|c|c|}
\hline \multirow[b]{2}{*}{ Predictors } & \multicolumn{3}{|c|}{$\begin{array}{l}\text { Correlation } \\
\text { coefficients }\end{array}$} & \multicolumn{3}{|c|}{$\begin{array}{l}\text { Standardized } \\
\text { coefficients }\end{array}$} \\
\hline & All & FTM & MTF & All & FTM & MTF \\
\hline Body protection & .917 & .935 & .817 & .766 & .780 & 698 \\
\hline Body image feelings and attitudes & .622 & .582 & .723 & .348 & .225 & .605 \\
\hline Body care & 498 & .518 & .373 & .087 & .090 & .064 \\
\hline Comfort in touch & .428 & .500 & .279 & .090 & .187 & -.122 \\
\hline
\end{tabular}

Note. $\quad$ FTM $=$ female-to-male; $\mathrm{MTF}=$ male-to-female.

\section{Standardized Coefficients and Correlations of Predictor Variables}

We were able to successfully classify $71.4 \%$ of the participants in the total sample as either having or not having a lifetime prevalence of NSSI based on their BIS score. To take into account chance agreement, we computed a kappa coefficient and obtained a moderate value of 0.39. To assess how well the classification procedure would statistically predict in a new sample, we estimated the percent of participants accurately classified using the leave-one-out technique 
and correctly classified $70.8 \%$ of participants. We then examined classification for just the FTM and MTF subsamples. Statistical prediction was better for MTF individuals (74.8\%) than it was for FTMs $(67.9 \%)$. Body protection remained the strongest predictor of lifetime NSSI for FTMs $(r=.935)$ and MTFs $(r=.817)$, Body image and feelings and attitudes represented a stronger statistical predictor among MTFs $(r=.723)$ than it was for FTMs $(r=.582)$; however, both of these results were statistically significant (Table 6).

\section{Discussion}

Using a large, online, convenience sample of TGNC adults in the United States, this study found that $41.9 \%$ of participants reported a history of NSSI (the original dataset includes people from other countries; we used only those from the United States for this study). This estimate is greater than most reported studies of NSSI in both clinical (20\% of adult psychiatric patients) and nonclinical samples (4\% of adults; Klonsky \& Muehlenkamp, 2007). It is important to consider the possibility that this number is inflated. Participants were given basic information about the purpose of the study on the informed consent page. It is possible that prospective participants read the general information about the survey, and since they did not have a history of NSSI, they chose not to continue with the survey. Despite this limitation, it is clear that many TGNC people have a history of NSSI, and therefore the topic of NSSI in the TGNC community warrants future clinical and research attention.

Participants with a history of NSSI showed higher levels of depression, anxiety, and stress than participants without a reported history of NSSI. It may be the case for these individuals that NSSI is a means of coping with these negative emotional experiences. Without further study, including mixed-methods research, it is difficult to know how these emotional experiences relate to a person's NSSI history. Specifically, these individuals may experience higher levels of negative emotion because of the challenges they face as a TGNC person in the U.S. where there are few legal protections for TGNC people (Taylor, 2007).

The mean age for NSSI initiation was 13 years in this sample, which is similar to other reports of NSSI starting in adolescence (Klonsky \& Muehlenkamp, 2007). However, the current study found that the self-reported occurrence of NSSI in our sample continued well into adulthood, which may be different from the usual course of NSSI in the general population. It is unclear from the cross-sectional nature of this study whether TGNC individuals follow a similar developmental trajectory than cisgender persons regarding the age at which they start and stop engaging in NSSI.

Participants in our study reported engaging in a similar variety and scope of NSSI as in previous studies among cisgender persons, with cutting as the most common behavior (Klonsky, 2007). One behavior that notes attention is the fact that over $25 \%$ of participants indicated sticking themselves with needles. Klonsky and Olino (2008) found a lifetime prevalence of sticking oneself with needles to be $13 \%$. Given the fact that many TGNC people have ready access to needles, which are sometimes used for cross-sex hormone administration, this type of NSSI may be more specific to this population. However, it is important to note that we did not assess the 
use of injectable hormone use in this sample. Further research is needed to understand how gender identity intersects with the method of NSSI a person uses.

Some members of the TGNC community may be at greater risk of lifetime NSSI. When considering gender, genderqueer individuals reported the highest lifetime prevalence of NSSI (51.6\%) followed by FTMs (45.5\%), other (nonbinary) participants (42.6\%), and MTF individuals with the lowest prevalence $(34.0 \%)$. When considering sexual orientation, people who identified as queer reported the highest rates of NSSI (62.3\%), followed by other sexual orientation (46.8\%), bisexual (45.1\%), asexual (38.5\%), lesbian/gay (36.7\%), and heterosexual $(26.4 \%)$. It should be noted that the numbers of people in the genderqueer and queer groups were small, and caution is warranted in assuming that the higher reported rates of NSSI are generalizable to these population. Even so, the reported rates of NSSI for genderqueer and queer individuals are much higher than the average for the full sample, which was $42.1 \%$. It is possible that lifetime prevalence estimates of NSSI are truly higher for people identifying as genderqueer and queer, especially as one considers the possible interaction of minority intersecting identities (Hendricks \& Testa, 2012). Furthermore, people who do not live within the gender binary, or are not heteronormative, are often mistreated in ways that might increase the level of subjective stress one experiences on a day in and day out basis, and such stress may then contribute to higher rates of NSSI.

Body image and protection of one's body are integral aspects of TGNC people's lives. Providers who work with individuals at risk for NSSI, or who have a history of NSSI behaviors, are encouraged to pay special attention to the need to work with clients to develop strengths-based coping strategies (American Counseling Association [ACA], 2010). For example, providers might work with clients to develop mindfulness skills to help the TGNC client work through their emotions without engaging in NSSI. Having a history of NSSI was also associated with higher self-reported levels of current depression, anxiety, and stress. Gaining a clearer understanding of functions of NSSI in the TGNC community, with attention to gender affirmation, may help to explain this finding and inform clinical work to meet the unique needs of TGNC people.

A lack of investment in one's body, as measured by the Body Investment Scale was statistically predictive of NSSI. Specifically, the subscales of Body Protection and Body Image Feelings and Attitudes may be useful in determining who may be at greater risk of or be more likely to have a history of NSSI. Mental health providers are encouraged to inquire about a client's investment in self-care and other ways a person might protect their body from harm. Previous research has shown that TGNC people are at risk of managing stress through the use of potentially harmful behaviors (Hepp et al., 2005). Future research is needed to determine exactly how the BIS might be used in a clinical setting.

\section{Limitations}

Several limitations are important to consider in the context of interpreting this study's findings. Survey research is often limited by the problems that are associated with self-reporting. Selfreport surveys may be completed in a manner to either inflate or diminish the overall affect of 
the queried behavior (e.g., recall bias). Related to the limitations regarding self-report are concerns about how participants made meaning of the question "Have you ever engaged in selfinjurious behavior (e.g., cutting, burning, hitting, pulling hair, etc.)?” Although the meaning of this question was clear in the minds of the researchers, it may not have been so for the research participants. As a result, a person who has, for example, pulled their hair one or more times may have been listed as having engaged in NSSI when that was not the intention behind their history of hair pulling. Another limitation is the use of an Internet-based convenience sample, which may limit the generalizability of findings. The variety of sources used for recruitment, which cannot be separated out, may also have an impact on the findings.

As can be seen from the demographics of the sample, this is not a representative sample as it is predominantly White (non-Hispanic) and highly educated. In addition, the sample of participants used in this study included more FTM than MTF individuals. It is generally accepted that there are more MTF people than there are FTM (Meier \& Labuski, 2013). The primary investigator and first author of the paper identifies as FTM and it is possible that more trans masculine individuals than trans feminine individuals responded affirmatively to his recruitment procedures. However, despite limitations, the strengths of the Internet convenience sampling approach include minimizing social desirability bias in the self-reporting of sensitive and potentially stigmatizing NSSI behaviors and being able to recruit a relatively large sample of TGNC people that allowed for powered statistical analyses. In spite of these limitations, researchers have supported the use of the Internet for data collection especially with communities who can be hard-to-reach (Reisner et al., 2014). Another demographic limitation of the study is the qualifying question in which participants were eligible if they identified as TGNC. This is considered by some to be an umbrella term for a broad group of people whose sex assigned at birth and gender identity or expression are not congruent; there are others who do not identify as TGNC and therefore may have self-selected out of the study (Grant et al., 2011).

\section{Implications}

The results of this study have implications for practice and research. The first is the need for providers to work closely with TGNC clients to assure that they have developed coping strategies that are likely to alleviate stress associated with being TGNC. This is not to say that a TGNC person should not be allowed to move forward in gender transition if they are using maladaptive coping strategies. Providers who are working with clients who seek a letter of support for transition (hormones or surgery) are encouraged to attend to the coping strategies in which a client engages, including screening for past and current NSSI. If a provider has concern about the client's readiness for transition and is not yet ready to write a letter of support, it would be important to assess the extent to which the client can utilize strengths-based coping strategies. This might include having ready access to a supportive community and use of self-soothing techniques (e.g., exercise and meditation) that are not inherently dangerous (e.g., NSSI, alcohol, and drug use). For some TGNC people, the need to ask for letters of support can create a difficult barrier in their realization of their gender (Lev, 2004). Providers are cautioned to avoid taking the role of gatekeeper with their clients. Lifetime history of NSSI does not necessarily preclude TGNC people from socially, medically, or legally affirming their gender. The current system that 
may require a letter of support from a psychologist addressing co-occurring mental health concerns can lead to tension for the psychologist. In this way, the psychologist may feel as though they are in a bind. On the one hand, they want to be supportive of their client and continue to build a positive therapeutic relationship. On the other hand, they may hold (withhold) permission in the form of a letter that will allow the client to realize their gender. Readers are referred to the World Professional Association for Transgender Health's Standards of Care for a complete discussion of the need for letters of support (Coleman et al., 2012).

Researchers are encouraged to consider the interaction between exploration of gender identity and history of NSSI. Longitudinal studies are best suited to understand the order in which these events occur. Psychologists wishing to develop competency in TGNC mental health are encouraged to view gender transition as a continuum and gender affirmation as a process with social, medical, and legal dimensions. Further research using prospective, longitudinal designs represents an important next step to understand how and when maladaptive coping strategies are used and what happens to the use of these strategies as a person moves forward in the gender transition process.

The current research can serve as the basis for additional research to increase understanding for when NSSI is or is not a contraindication to gender transition. Empirically supported interventions that offer TGNC people opportunities to foster positive stress-coping behaviors are warranted. Finally, the development of a risk assessment that is geared specifically for TGNC people could be used to assist clinicians in their work with TGNC clients. Such a tool needs to be flexible enough to capture the differences in a client's gender identity, which may shift over time (ACA, 2010). As the language that TGNC people use to identify themselves often shifts, it is important that the tool reflect this fluidity.

\section{Conclusion}

This study was designed to assess the lifetime prevalence of NSSI in a large, online sample of TGNC adults in the United States. Nearly $42 \%$ of respondents had a history of NSSI. This behavior was more common in FTMs than in MTFs. TGNC people face many challenges in life. Often they are faced with discriminative work environments, hostile families and friends, and underemployment. Working with TGNC people to employ positive coping strategies in managing stressors represents an important therapeutic goal. Screening for past and/or current NSSI is recommended. TGNC people are deserving of compassionate and competent care. The current study may provide professionals with a keener insight to NSSI, a clinical issue that affects some members of the TGNC community. 


\section{References}

American Counseling Association. (2010). Competencies for counseling with transgender clients. Journal of LGBT Issues in Counseling, 4, 135-159.

Association of Lesbian, Gay, Bisexual, and Transgender Issues in Counseling. (2009). Competencies for counseling with transgender clients. Alexandria, VA: Author.

Clements-Nolle, K., Marx, R., \& Katz, M. (2006). Attempted suicide among transgender persons: The influence of gender-based discrimination and victimization. Journal of Homosexuality, 51, 53-69. 10.1300/J082v51n03_04

Coleman, E., Bockting, W., Botzer, M., Cohen-Kettenis, P., DeCuypere, G., Feldman, J., . . .Zucker, K. (2012). Standards of care for the health of transsexual, transgender, and gender nonconforming people, 7th version. International Journal of Transgenderism, 13, 165-232. 10.1080/15532739.2011.700873

Favazza, A. R. (1998). The coming of age of self-mutilation. Journal of Nervous and Mental Disease, 186, 259-268. 10.1097/00005053-199805000-00001

Grant, J. M., Mottet, L. A., Tanis, J., Harrison, J., Herman, J. L., \& Keisling, M. (2011). Injustice at every turn: A report of the National Transgender Discrimination Survey. Washington, DC: National Center for Transgender Equality \& National Gay and Lesbian Task Force.

Hendricks, M. L., \& Testa, R. J. (2012). A conceptual framework for clinical work with transgender and gender nonconforming clients: An adaptation of the minority stress model. Professional Psychology: Research and Practice, 43, 460-467. $10.1037 / \mathrm{a} 0029597$

Henry, J. D., \& Crawford, J. R. (2005). The short-form version of the Depression Anxiety Stress Scales (DASS-21): Construct validity and normative data in a large non-clinical sample. British Journal of Clinical Psychology, 44, 227-239. $10.1348 / 014466505 \times 29657$

Hepp, U., Kraemer, B., Schnyder, U., Miller, N., \& Delsignore, A. (2005). Psychiatric comorbidity in gender identity disorder. Journal of Psychosomatic Research, 58, 259261. 10.1016/j.jpsychores.2004.08.010

Huberty, C. J. (1984). Issues in the use and interpretation of discriminant analysis. Psychological Bulletin, 95, 156-171. 10.1037/0033-2909.95.1.156

Institute of Medicine. (2011). The health of lesbian, gay, bisexual, and transgender people: Building a foundation for better understanding. Washington, DC: National Academies Press.

Jacobson, C. M., \& Gould, M. (2007). The epidemiology and phenomenology of non-suicidal self-injurious behavior among adolescents: A critical review of the literature. Archives of Suicide Research, 11, 129-147. 10.1080/13811110701247602 
Klonsky, E. D. (2007). The functions of deliberate self-injury: A review of the evidence. Clinical Psychology Review, 27, 226-239. 10.1016/j.cpr.2006.08.002

Klonsky, E. D., \& Glenn, C. R. (2009). Assessing the functions of non-suicidal self-injury: Psychometric properties of the Inventory of Statements About Self-injury (ISAS). Journal of Psychopathology and Behavioral Assessment, 31, 215-219. $10.1007 / \mathrm{s} 10862-008-9107-\mathrm{z}$

Klonsky, E. D., \& Muehlenkamp, J. J. (2007). Self-injury: A research review for the practitioner. Journal of Clinical Psychology, 63, 1045-1056. 10.1002/jclp.20412

Klonsky, E. D., \& Olino, T. M. (2008). Identifying clinically distinct subgroups of self-injurers among young adults: A latent class analysis. Journal of Consulting and Clinical Psychology, 76, 22-27. 10.1037/0022-006X.76.1.22

Lambda Legal. (2008). Bending the mold: An action kit for transgender students. Retrieved from [http://data.lambdalegal.org/publications/downloads/btm_bending-the-mold.pdf]

Latimer, S., Meade, T., \& Tennant, A. (2013). Measuring engagement in deliberate self-harm behaviours: Psychometric evaluation of six scales. BMC Psychiatry, 13, 4. 10.1186/1471244X-13-4

Lev, A. I. (2004). Transgender emergence: Therapeutic guidelines for working with gendervariant people and their families. Binghamton, NY: Haworth Clinical Practice.

Lovibond, S. H., \& Lovibond, P. F. (1995). Manual for the Depression Anxiety Stress Scales. Sydney, Australia: University of New South Wales.

Meier, S. C., \& Labuski, C. M. (2013). The demographics of the transgender population. In A. K.Baumle (Ed.), International handbook of the demography of sexuality (pp. 289-327). New York, NY: Springer. 10.1007/978-94-007-5512-3_16

Muehlenkamp, J. J. (2005). Self-injurious behavior as a separate clinical syndrome. American Journal of Orthopsychiatry, 75, 324-333. 10.1037/0002-9432.75.2.324

Nock, M. K. (2009). Why do people hurt themselves? New insights into the nature and functions of self-injury. Current Directions in Psychological Science, 18, 78-83. 10.1111/j.14678721.2009.01613.x

Orbach, I., \& Mikulincer, M. (1998). The body investment scale: Construction and validation of a body experience scale. Psychological Assessment, 10, 415-425. 10.1037/10403590.10 .4 .415

Reisner, S. L., Conron, K., Scout, N. F. N., Mimiaga, M. J., Haneuse, S., \& Austin, S. B. (2014). Comparing in-person and online survey respondents in the U.S. National Transgender Discrimination Study: Implications for transgender health research. LGBT Health, 1, 98106. 
Sánchez, F. J., \& Vilain, E. (2009). Collective self-esteem as a coping resource for male-tofemale transsexuals. Journal of Counseling Psychology, 56, 202-209. 10.1037/a0014573

Taylor, J. K. (2007). Transgender identities and public policy in the United States: The relevance for public administration. Administration \& Society, 39, 833-856. 10.1177/0095399707305548

Vocks, S., Stahn, C., Loenser, K., \& Legenbauer, T. (2009). Eating and body image disturbances in male-to-female and female-to-male transsexuals. Archives of Sexual Behavior, 38, 364-377. 10.1007/s 10508-008-9424-z 